\title{
Periodic Target Accrual Number
}

National Cancer Institute

\section{Source}

National Cancer Institute. Periodic Target Accrual Number. NCI Thesaurus. Code C93621.

A range of integers specifying the minimum and maximum number of subjects to be accrued per a specified amount of time. 